\title{
The mass of a halo
}

\begin{abstract}
M. White
Harvard-Smithsonian Center for Astrophysics, Cambridge, MA 02138, USA

e-mail: mwhite@cfa.harvard.edu

Received 1 September 2000 / Accepted 17 November 2000

Abstract. We discuss the different definitions of the mass of a halo in common use and how one may convert between them. Using $N$-body simulations we show that mass estimates based on spherical averages are much more tightly correlated with each other than with masses based on the number of particles in a halo. The mass functions pertaining to some different mass definitions are estimated and compared to the "universal form" of Jenkins et al. (2000). Using a different simulation pipeline and a different cosmological model we show that the mass function is well fit by the Jenkins et al. (2000) fitting function, strengthening the claim to universality made by those authors. We show that care must be taken to match the definitions of mass when using large $N$-body simulations to bootstrap scaling relations from smaller hydrodynamical runs to avoid observationally significant bias in the predictions for abundances of objects.
\end{abstract}

Key words. methods: $N$-body simulations

\section{Introduction}

One of the most fundamental predictions of a theory of structure formation is the number density of objects of a given mass, the mass function, at a given redshift. Accurate mass functions are used in a number of areas in cosmology; in studies of galaxy formation, in measures of volumes (e.g. galaxy lensing) and in attempts to infer the normalization of the power spectrum and the density parameter from the abundance of rich clusters. In the latter case the mass function is the point of contact allowing us to bootstrap the excellent statistics of large $N$-body simulations with observable properties of clusters normalized for example by hydrodynamic simulations of smaller volumes. In this way we can obtain reliable estimates of e.g. the number of clusters as a function of temperature and redshift, which can in turn be used to constrain the matter density, the normalization of the power spectrum and the statistics of the initial density field.

Several different definitions for the "mass" of a halo are in common use, each having different advantages. For example there are at least 3 different definitions of mass for the mass-temperature relation as computed from hydrodynamical simulations of galaxy clusters and all are different from the definition of mass commonly used in the mass function which is itself different from the mass usually employed in analytic studies based on the PressSchechter (1974; hereafter PS) theory. Observational data have improved to the point where it is important to distinguish between these different definitions of mass, lest we bias our theoretical predictions. We give an example of how such a conversion can be made, at least approximately, for a certain class of mass estimator.

\section{Halo mass definitions}

In this section we describe some of the definitions of the mass of a dark matter halo in common use. This list is obviously not exhaustive, but it is representative. We begin by recalling some background about the spherical top-hat collapse model (see e.g. Peebles 1993; Peacock 1999; Liddle \& Lyth 2000, and references therein) from which much of the language in this field has been borrowed.

The spherical top-hat ansatz describes the formation of a collapsed object by solving for the evolution of a sphere of uniform overdensity $\delta$ in a smooth background of density $\bar{\rho}$. By Birkhoff's theorem the overdense region evolves as a positively curved Friedman universe whose expansion rate is initially matched to that of the background. The overdensity at first expands but because it is overdense the expansion slows (relative to the background) and eventually halts before the region begins to recollapse. Technically the collapse proceeds to a singularity but it is assumed in a "real" object virialization occurs at twice $^{1}$ the turn-around time, resulting in a sphere of half the turn-around radius. In an Einstein-de Sitter model the overdensity (relative to the critical density) at virialization is $\Delta_{\mathrm{c}}=18 \pi^{2} \simeq 178$. We shall always use $\Delta_{\mathrm{c}}$ to indicate the overdensity relative to critical of a virialized halo, which will be lower for smaller $\Omega_{\mathrm{M}}$. Note that some authors use a

\footnotetext{
1 There is a small correction to this in the presence of a cosmological constant which contributes a $\Lambda r^{2}$ potential.
} 
different convention in which $\Delta_{\mathrm{c}}$ is specified relative to the background matter density - our $\Delta_{c}$ is $\Omega_{\mathrm{M}}$ times theirs. The linear theory extrapolation of this overdensity is normally denoted $\delta_{\mathrm{c}}$ and is $(3 / 20)(12 \pi)^{2 / 3} \simeq 1.686$ in an Einstein-de Sitter model. This overdensity is often used as a threshold parameter in PS theory and its extensions and has a very weak cosmology dependence which is often neglected.

We now turn to some of the definitions of mass in use in the literature. Let us suppose that we have identified some object, a problem to which we shall return in Sect. 4, and have chosen a fiducial "center" about which to take spherically averaged profiles. We define $M_{\Delta}$ as the mass contained within a radius $r_{\Delta}$ inside of which the mean interior density is $\Delta$ times the critical density

$\int_{0}^{r_{\Delta}} r^{2} \mathrm{~d} r \rho(r)=\frac{\Delta}{3} \rho_{\text {crit }} r_{\Delta}^{3}$.

The "virial mass" from the spherical top-hat collapse model would then be simply $M_{\Delta_{c}}$. We shall refer to this mass as $M_{\mathrm{th}-\mathrm{vir}}$.

An alternative interpretation of the spherical collapse model is that the virial radius corresponds to that point within which the material is virialized and external to which the mass is still collapsing onto the object. Some simulations suggest that this occurs at $\Delta=200$ more or less independent of cosmology, and so a common mass estimator is $M_{200}$ which is approximately the virial mass if $\Omega_{\mathrm{M}}=1$. Authors also use $M_{500}$ or $M_{1000}$ in some applications.

The final mass estimator we shall consider is the one used by Jenkins et al. (2000) in their analysis of simulations performed by the Virgo consortium. The mass they assign to a halo is simply the sum of the masses of the particles identified as members of the halo by their halo finder. This mass estimator is the least easily interpreted theoretically, though it is well defined algorithmically, but has the very convenient feature that the mass function for this estimator is independent of cosmology!

Unfortunately there is no unique algorithmic definition of a dark matter halo, even within a 3D simulation itself. To define the mass of an object we must first specify the object in question! Although other halo finders are in common use, we shall deal exclusively with halos found using the Friends-of-Friends (Davis et al. 1985) algorithm, hereafter called FOF. Our reason for this choice is that it is the group finder employed by the Virgo consortium in deriving the mass functions from their very large $N$-body simulations (Jenkins et al. 2000) and in terms of which they find a universal mass function. The FOF algorithm has one free parameter, $b$, the linking length in units of the mean inter-particle spacing. Commonly used values of $b$ are $0.1,0.15$ and 0.2 , although other choices exist (e.g. Gardner 2000 chooses instead $b^{-3}=\Omega_{\mathrm{M}} \Delta_{\mathrm{c}} / 3$ ). Jenkins et al. (2000) find that the mass function is universal if they take $b=0.2$, independent of the cosmology under consideration. Jenkins et al. (2000) claim that in the limit of a large number of particles per halo, FOF finds

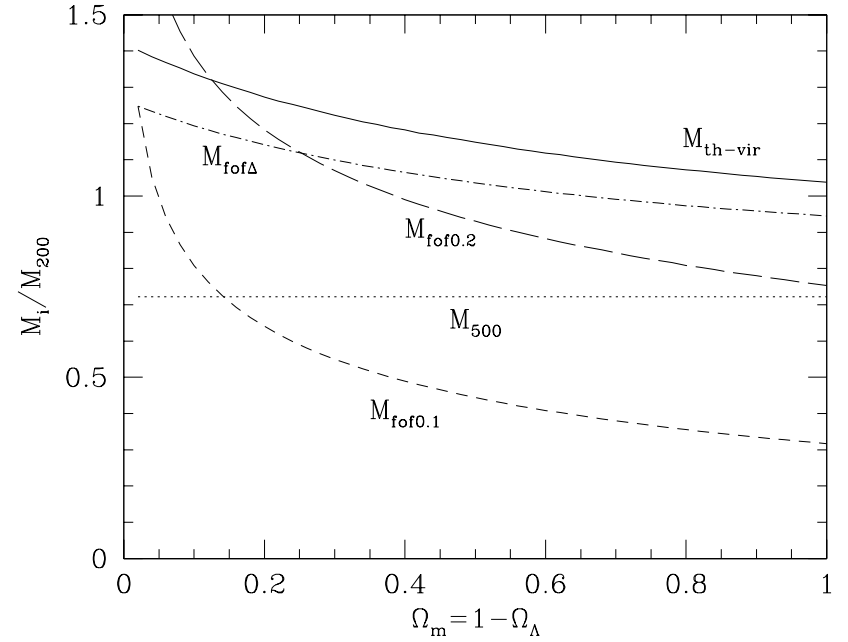

Fig. 1. The relations between various definitions of the mass of a halo as a function of $\Omega_{\mathrm{M}}$ assuming the halo density profile follows the NFW form with concentration parameter $c=5$

all particles within an iso-density contour $b^{-3}$ times the mean matter density or $\Omega_{\mathrm{M}} / b^{3}$ times critical density. We shall return to this point in Sect. 4 .

In the next sections we describe how these different masses are related.

\section{An approximate conversion}

It would be advantageous if we could convert, at least approximately, between the different definitions of mass in common use. In this section we make a first attempt in this direction. We assume that all halos are spherical and have a universal profile, for example ${ }^{2}$ the NFW form (Navarro et al. 1996):

$\rho(r) \propto x^{-1}(1+x)^{-2}$,

where $x=r / r_{\mathrm{s}}$ and $r_{\mathrm{s}}$ is a scale radius usually specified in terms of the concentration parameter $c \equiv r_{200} / r_{\mathrm{s}}$. Navarro et al. (1996) refer to $r_{200}$ and $M_{200}$ throughout as the "virial radius" and "virial mass" respectively. Again, $N$-body simulations have shown that the concentration parameter is a weak function of virial mass, taking the value $c \sim 5$ for masses characteristic of clusters which shall be our focus here.

With a given profile it is straightforward to relate the various mass definitions as shown in Fig. 1.

Note that the masses can differ significantly in this model and that the cosmology dependence is quite strong. How well does this crude model predict the relationship between different mass definitions in practice?

2 There is some controversy in the literature about the slope of the density profile as $r \rightarrow 0$. Luckily departures from the NFW form are expected to occur only at very small radii, containing a vanishingly small amount of the total mass, and thus do not concern us here. 
Table 1. Correlations between the various mass definitions for the halos of our large simulation. Only halos with more than 1000 particles are used

\begin{tabular}{c|ccc}
\hline & $M_{\text {fof } 0.2}$ & $M_{\text {th-vir }}$ & $M_{200}$ \\
\hline$M_{\text {fof } 0.2}$ & 1.000 & 0.974 & 0.966 \\
$M_{\text {th-vir }}$ & 0.974 & 1.000 & 0.997 \\
$M_{200}$ & 0.966 & 0.997 & 1.000 \\
\hline
\end{tabular}

\section{Simulations}

The model of the previous section made several approximations. To compare these different mass estimates with each other more carefully we have used some $N$-body simulations, originally run for another purpose. We simulated the Ostriker \& Steinhardt (1995) concordance model, which has $\Omega_{\mathrm{m}}=0.3, \Omega_{\Lambda}=0.7, H_{0}=100 h \mathrm{~km} \mathrm{~s}^{-1} \mathrm{Mpc}^{-1}$ with $h=0.67, \Omega_{\mathrm{B}}=0.04, n=1$ and $\sigma_{8}=0.9$ (corresponding to $\left.\delta_{\mathrm{H}}=5.0210^{-5}\right)$. For this cosmology $\Delta_{\mathrm{c}} \simeq 101$ from the top-hat collapse model.

We have used two "high" resolution and two lower resolution $N$-body simulations. The first was a $256^{3}$ particle, dark matter only simulation in a $100^{3} h^{-1} \mathrm{Mpc}$ box. The simulation was run on 16 processors of the Origin2000 at NCSA with the TreePM-SPH code (White et al. 2000) operating in collisionless (dark matter only) mode. The gravitational force softening was of a spline form (e.g. Hernquist \& Katz 1989), with a "Plummerequivalent" softening length of $15 h^{-1} \mathrm{kpc}$ comoving. The simulation was evolved from $z=100$ until the present and took a total of 1300 (normalized) CPU hours. A second $256^{3}$ particle simulation was run from $z=70$ in a $256 h^{-1}$ Mpc box with a $35 h^{-1} \mathrm{kpc}$ softening. This run took a total of 880 (normalized) CPU hours, also on 16 processors. To provide additional statistics on the highmass end of the mass function we additionally ran several smaller $\left(128^{3}\right.$ particles $)$ simulations of the same model in boxes of side $200 h^{-1}$ Mpc.

From the $z=0$ outputs we generated group catalogues using the FOF algorithm with $b=0.2$. For each halo we defined the center for our spherical averages as the particle with the minimum potential energy. This corresponds closely to the most bound particle and the density peak for a halo in all but the most disturbed systems, and is more robust than the center of mass. With the group catalog and centers so defined it is straightforward to calculate each of the estimators described above. Throughout we shall use $M_{200}$ as our "base" mass to which the others are compared, since this fits best into the philosophy of Sect. 3 .

We show in Fig. 2 that the different mass estimators are indeed highly correlated as one would expect (see also Table 1).

The ratio of the FOF mass to $M_{200}$ is seen to span a reasonable range and be offset from unity by a nonnegligible amount. Figure 3 shows a histogram of the ratio of $M_{\mathrm{fof} 0.2}$ and $M_{\mathrm{th} \text {-vir }}$ to $M_{200}$ for all of the halos above a given number of particles.
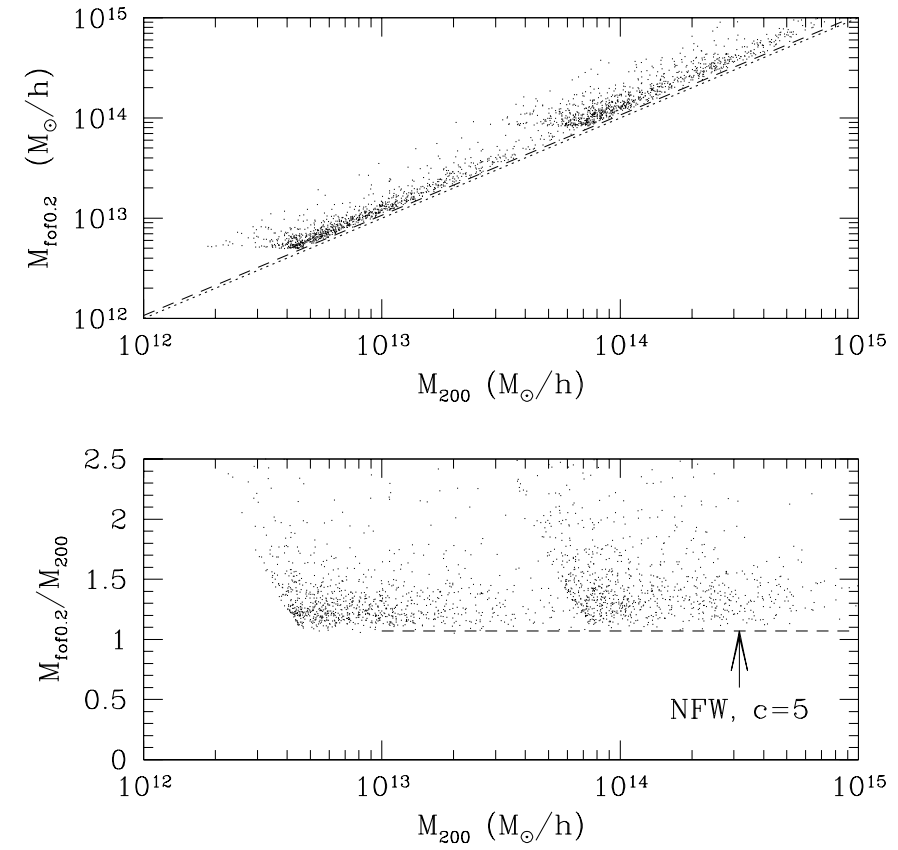

Fig. 2. Correlations between two estimators of the mass, $M_{\text {fof } 0.2}$ and $M_{200}$, in simulations of a $\Lambda$ CDM model (see text). Only halos with more than 1000 particle are used. The upper panel shows that the different estimates of mass are correlated, but offset (the dotted line marks $y=x$ ). The dashed line indicates the prediction for the offset of Sect. 3. The lower panel shows the mass ratios as a function of $M_{200}$ on a linear scale

Let us focus first on $M_{\mathrm{th} \text {-vir }}$. The shape of the histogram is roughly the same regardless of particle number indicating that the distribution is not strongly affected by finite particle numbers in the halos chosen. The tophat virial mass and $M_{200}$ are fairly tightly correlated, as one might expect since they are both spherically averaged statistics and the density contrasts are not too different. The approximation of Sect. 3 provides a reasonable estimate of the ratio of masses, presumably because of the spherical averaging being performed.

The situation with regards $M_{\text {fof } 0.2}$ is more complicated. Firstly the scatter is much larger, since the groups found by FOF can be quite irregularly shaped (see Fig. 4). The actual ratio of $M_{\text {fof } 0.2}$ to $M_{200}$ is larger than predicted by our model. By eye the halo finder looks to have merged close groups. It is possible that with better mass resolution this would occur to a lesser extent, although the lack of a clear trend with particle number in Fig. 3 argues that convergence would be quite slow.

To investigate whether FOF has merged adjacent halos and whether a FOF halo can be considered as all particles above a given density threshold (as assumed in Sect. 3) we have examined the particles in the two groups of Fig. 4. For each particle we calculate its distance from the halo 


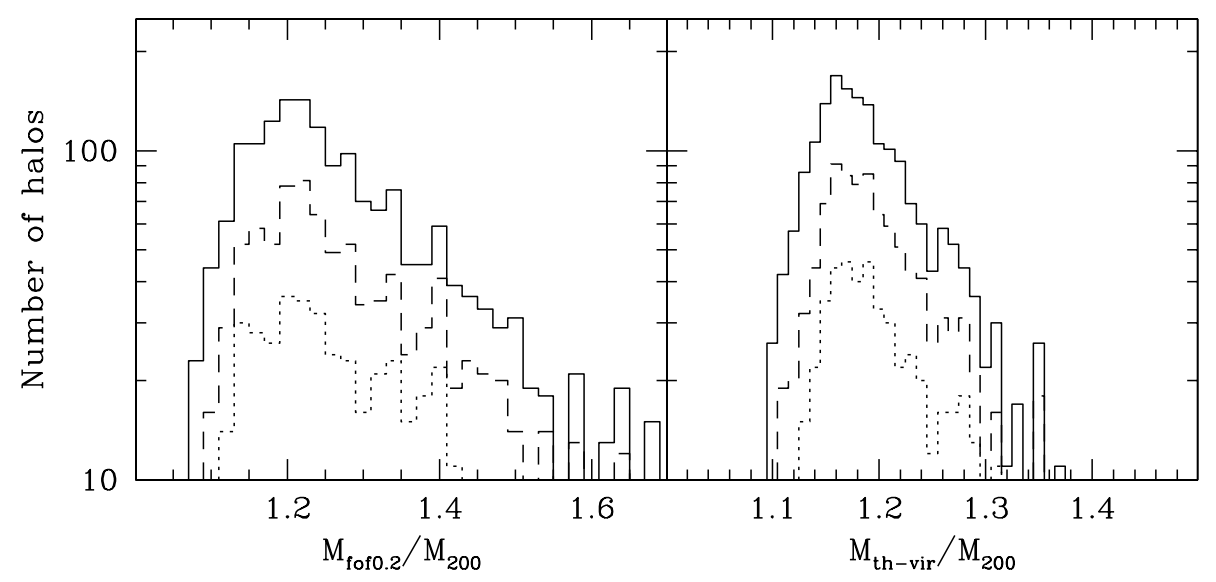

Fig. 3. Distribution of mass ratios for halos from the first simulation. The solid line shows all halos with more than 500 particles, the dashed line 1000 particles and the dotted line 2000 particles. The ratios predicted by the model of Sect. 3 are $M_{\mathrm{fof} 0.2} / M_{200}=$ 1.07 and $M_{\mathrm{th}-\mathrm{vir}} / M_{200}=1.22$
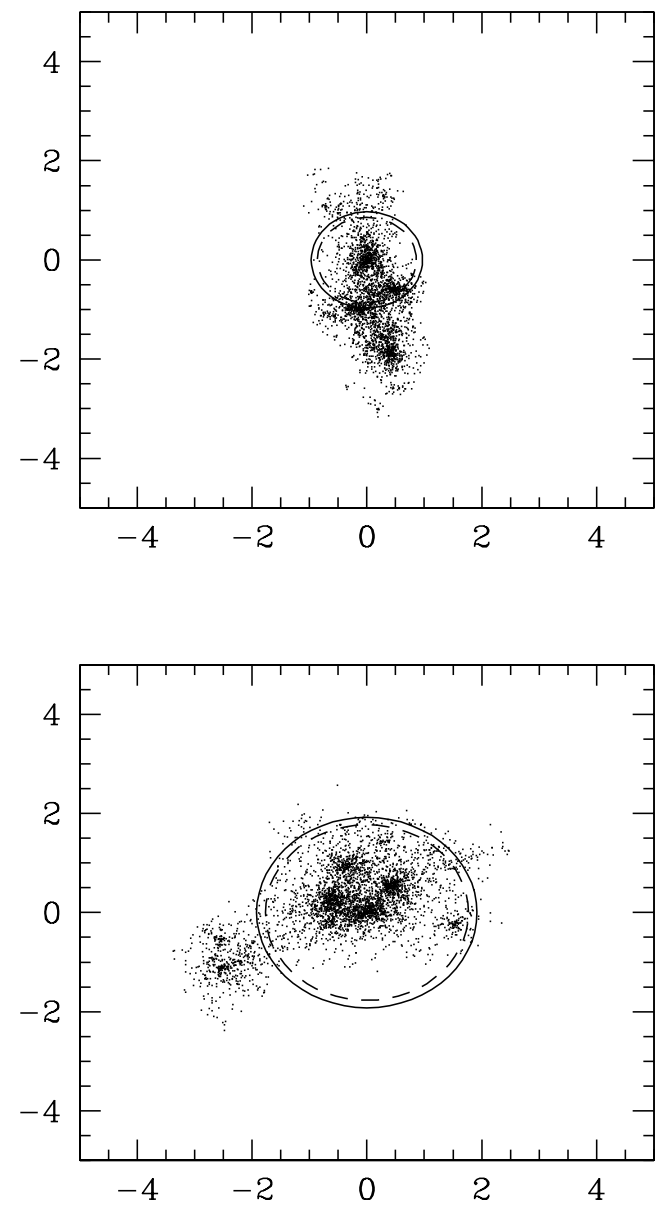

Fig. 4. The mass distribution for two groups from the first simulation, along with the top-hat "virial" radius (solid) and $r_{200}$ (dashed line). An $x-y$ projection, with axes in $h^{-1} \mathrm{Mpc}$, is shown centered on the point of minimum potential. The upper panel shows a group with $M_{200} \simeq 10^{14} h^{-1} M_{\odot}(62000$ particles; $5 \%$ shown), while the lower panel shows the largest group in the simulation $\left(M_{200}=810^{14} h^{-1} M_{\odot}, 210^{5}\right.$ particles; $2 \%$ shown). We have plotted only a fraction of the points for clarity, this suppresses the appearance of substructure in the group. There is a tendency for the larger groups to be more irregular

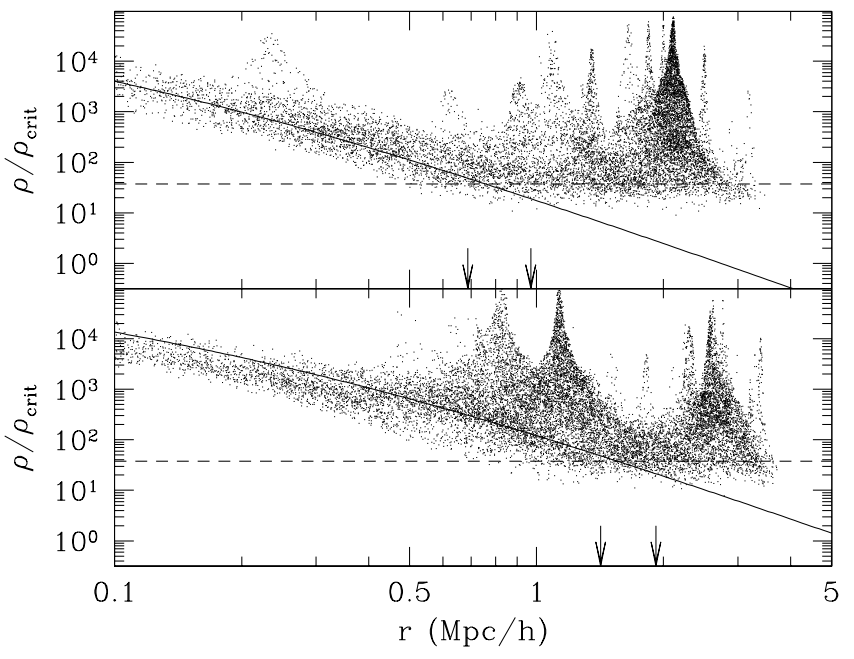

Fig. 5. The halo density profile, as a function of radial distance from the group center, for the groups of Fig. 4. Each dot represents a particle in the group (only a small fraction of the particles are shown for clarity) and the density is estimated through Eq. (3). The dashed horizontal line is $\Omega_{\mathrm{M}} b^{-3}$, the arrows mark $r_{200}$ and $r_{\text {th-vir }}$ and the solid line is the "model" NFW profile of Sect. 3, with $c=5$

center and its density, defined from the distance $\left(r_{\text {neig }}\right)$ to the 32 nd nearest neighbour ${ }^{3}$ as

$\rho \equiv \frac{3 M}{4 \pi} r_{\text {neigh }}^{-3}$

where $M$ is the enclosed mass. This density estimate is somewhat noisy, but will be sufficiently accurate for our purposes.

The results are shown in Fig. 5 along with the appropriate NFW profile (assuming $c=5$ ) for comparison. Note that the substructure in the halos is readily apparent as density "spikes" as functions of radius and can be matched to "sub-halos" in Fig. 4.

\footnotetext{
${ }^{3}$ We use all of the particles in the simulation, not just group members, in computing $r_{\text {neig }}$.
} 


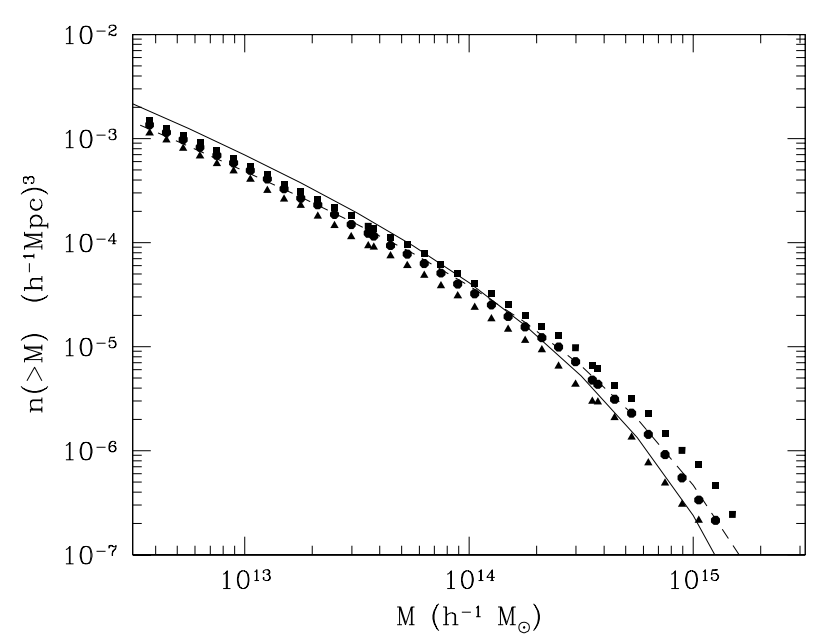

Fig. 6. The cumulative mass function for the Concordance model. The points are mass functions from the simulations where the mass is defined as: FOF0.2 (squares), $M_{\text {th-vir }}$ (circles) and $M_{200}$ (triangles). The solid line shows the predictions of the Press-Schechter theory, the dashed line is the fit of Jenkins et al. (2000)

It is clear from Fig. 5 that FOF is (at least approximately) linking all particles above a density $\Omega_{\mathrm{M}} b^{-3}$ of critical. However the chosen contrast is low enough that this includes particles well outside $r_{200}$ (or the virial radius) and the presence of these "nearby" particles is biasing the masses above $M_{200}$ and contributing to the extra scatter. This problem could be mitigated to some extent by reducing $b$, but it is not clear whether the mass function would remain universal if a different (cosmology independent) $b$ were chosen.

Finally we show in Fig. 6 the different mass functions that would be derived from these halos using the 3 different mass definitions. Note that our results, obtained with a completely independent $N$-body code and analyzed with a completely independent set of software, agree well with the universal mass function of Jenkins et al. (2000). However, especially at the high mass end, the number density is quite sensitive to the mass definition used. Intriguingly the fitting formula quoted by Jenkins et al. (2000) provides a good fit to the mass function if we use $M_{\mathrm{th} \text {-vir }}$ as our definition of mass, an even better fit than if we use $M_{\text {fof } 0.2}$.

\section{An example: The temperature function}

An example of where this mass uncertainty may be important is in using the observed temperature function of local X-ray clusters to constrain the normalization of the present day power spectrum. Although Pen (1998) has argued for a direct prediction of the temperature function from simulations, most people use a hybrid approach in obtaining this constraint. First a mass function, usually calibrated by simulations such as those of Jenkins et al. (2000), is assumed and then a mass temperature relation from hydrodynamic simulations is used to predict the temperature function.
Hydrodynamic simulations show good agreement for the total mass and X-ray temperature properties of clusters (e.g. Frenk et al. 1999). The mass-temperature relation from these simulations generally follows the virial relation quite well. Ignoring a small correction from the $\Lambda r^{2}$ potential, for an object virialized at a redshift $z$

$M(T, z) \propto[T /(1+z)]^{3 / 2}$

and the proportionality constant is then fixed by the simulations. There still remains some disagreement over the precise coefficient and its dependence on cosmology (see e.g. Henry 2000 for a recent compilation) but in addition to this different authors choose different conventions for $M$. For reasonable $\Omega_{\mathrm{M}}$, the difference in mass definitions is about the same size as the difference in proportionality constants between groups and the $1 \sigma$ scatter in $M$ at fixed $T$ found by the different groups. Unfortunately this alone does not account for all of the discrepancy.

To take only two relations as examples, Evrard (1996) define the mass in Eq. (4) as $M_{200}$. The $M$ of Bryan \& Norman (1998), on the other hand, is the "virial" mass in the sense of the spherical top-hat model. In both cases there is ample justification for the definition chosen, and the authors are explicit about their choice. However, there is an $\Omega_{\mathrm{M}}$ dependent ratio between these two definitions which cannot be neglected if the temperature function is to be predicted to the factor of 2 level!

\section{Conclusions}

We have shown that different definitions of the "mass" of a halo exist, and have different strengths and weaknesses. It is important to be consistent when combining relations which use different definitions of mass, and we have given an approximate method for converting between some commonly used mass estimators. Mass estimates based on spherical averages are much more tightly correlated with each other than with the mass obtained simply by summing the particles in the group, and can be quite well estimated by assuming a "universal" spherical profile (Sect. 3). The hydrodynamical simulations which calibrate observables as a function of cluster mass typically use such a spherically averaged mass definition.

Unfortunately these definitions are not very tightly correlated with the particle based mass used in the universal mass function of halos reported by Jenkins et al. (2000). We have argued that this is because, with a linking length of $b=0.2$, FOF is merging neighbouring halos. Such a problem would be mitigated by reducing $b$, but it has not been demonstrated that the mass function is universal for $b \neq 0.2$.

In the cosmological model we have simulated, the fitting form of Jenkins et al. (2000) provides a good match to the mass function of our halos, strengthening the claim of those authors that it is universal. However we find the best fit when the mass estimator used is the top-hat virial mass, rather than the FOF mass. While we have not investigated other cosmological models, we expect that the 
profiles (from the point of view of estimating masses) will not be very cosmology dependent. It would be interesting to see whether the Jenkins et al. (2000) mass function is "universal" if one uses the (cosmology dependent) top-hat virial mass of the halos.

Acknowledgements. I would like to thank V. Springel for the use of his FOF group finder, J. Mohr for useful conversations on this issue, and C. Metzler for encouraging me to write it up. M. White was supported by the US National Science Foundation and a Sloan Fellowship. Parts of this work were done on the Origin2000 system at the National Center for Supercomputing Applications, University of Illinois, UrbanaChampaign.

\section{References}

Bryan, G. L., \& Norman, M. L. 1998, ApJ, 495, 80

Davis, M., Efstathiou, G., Frenk, C. S., \& White, S. D. M. 1985, ApJ, 292, 371
Evrard, A. E., Metzler, C., \& Navarro, J. F. 1996, ApJ, 469, 494

Frenk, C. S., et al. 1999, ApJ, 525, 554

Gardner, J. P. 2000, preprint [astro-ph/0006342]

Henry, J. P. 2000, ApJ, in press [astro-ph/0002365]

Hernquist, L., \& Katz, N. 1996, ApJS, 70, 419

Jenkins, A., Frenk, C. S., White, S. D. M., et al. 2000, MNRAS, in press [astro-ph/0005260]

Liddle, A., \& Lyth, D. 2000, Cosmological Inflation and Large-Scale Structure (Cambridge University Press, Cambridge), 11

Navarro, J. F., Frenk, C. S., \& White, S. D. M. 1996, ApJ, 462, 563

Ostriker, J., \& Steinhart, P. J. 1995, Nature, 377, 600

Peacock, J. A. 1999, Cosmological Physics (Cambridge University Press, Cambridge), 15

Pen, U.-L. 1998, ApJ, 504, 601

Peebles, P. J. E. 1993 Principles of Physical Cosmology, (Princeton University Press, Princeton), chapter 25

Press, W. H., \& Schechter, P. 1974, ApJ, 187, 452

White, M., Springel, V., Coldwell, C., \& Hernquist, L., in preparation 